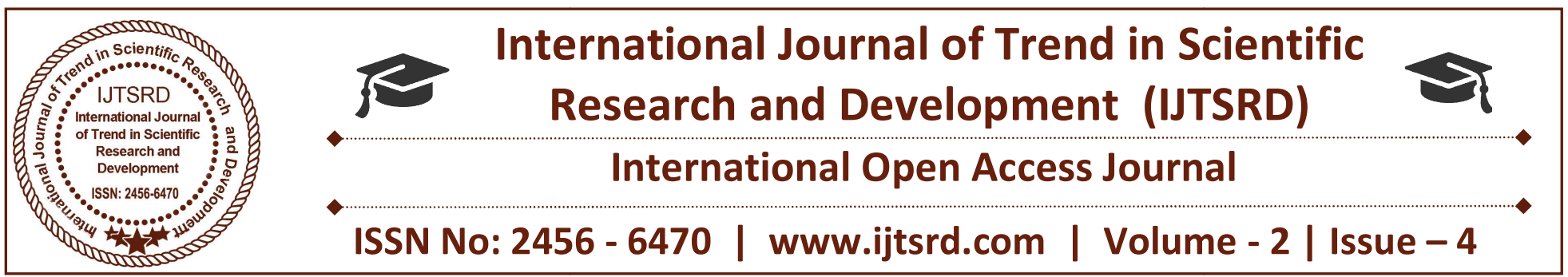

\title{
IoT Based Smart Clothing
}

\author{
Thejashwini. K. S, Sayeeqa Anjum, Sowmya K. N, C R Manjunath \\ Department of Information Science and Engineering, Jain University, Bangalore, India
}

\begin{abstract}
Smart clothing is on tremendous high demand for the various safety purposes, for heath in hazardous environment, for improvising business performance, appearance and in entertainment. With the wearable devices like smart jackets and other accessories it is possible to collect and detect the signals for the organizations to respond the incidents in many different ways.Smart Clothing joins in materials and remote sensor and organizes with regards to human body observing with measurable strategies for the information and treatment.
\end{abstract}

\section{INTRODUCTION}

In the electronics market consumers has accepted the wearable technology along with the high growth of technology of smart phones, tablets, laptops, and now it is poised to grow into the leader. Advanced circuitry and independent processing is the result of the devices that are worn on the body which can also be extended for any period of time.

The wearable devices are evolving in the new trend which helps in data analysis and communication about the body with the help of smart phones. Technologists are giving buyers more information about their bodies and lives than any other time ever by utilizing sensors, machine learning, and gig data analysis combination. This developing field will of products will have huge impact on human computer interaction.

The market for wearables has a few categories of individual devices, where every one of them are worn or appended to the body. The wearable technology can be classified and included in smart watches, body sensors, wearable cameras, location trackers, motion gadgets, and smart clothing. An extensive variety of purposes from human health care services to lifelogging, safety notifications are served by these devices. Smart clothing, or e- $\neg$ textiles, has fiber strands that are conductive or sensors connected to them or woven into the worn material. Like different wearables, smart clothing enables the consumer to assess the data that it sends information to secondary devices.

Health care is an exceptionally fundamental piece of life. Unfortunately, the fast growing population and the related perpetual disease is setting critical strain on present day medicinal services frameworks, and the interest for assets from doctor's facility beds to specialists and attendants is to a great degree high. Obviously, an answer is required to lessen the weight on health care solutions while proceeding to provide the care to the patient in danger.

The Internet of Things (IoT) has been broadly distinguished as a potential answer for ease the weights on health care solutions and it is the solution to carry out the research in recent trends. A lot of this exploration has been done at observing patients with particular conditions, for example, diabetes or Parkinson's infection. Additionally researches hope to fill particular needs, for example, aiding rehabilitation through constant monitoring of a patient's progress. The research is still ongoing process for these types of related works during the emergency health care. 


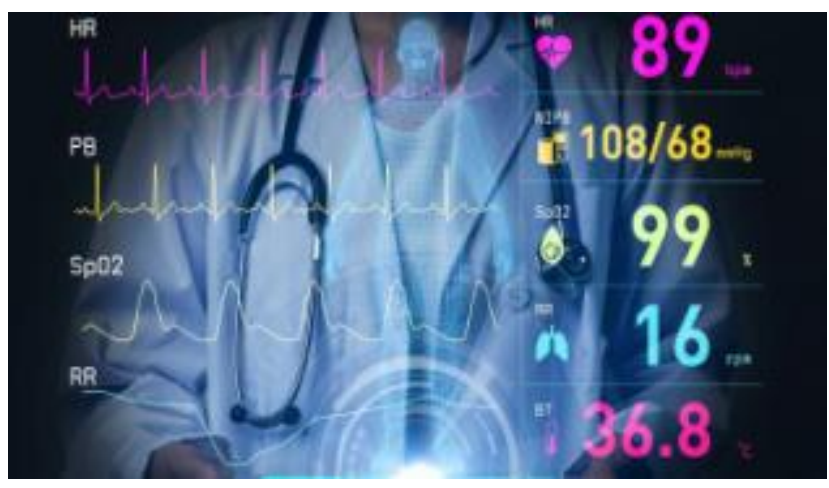

Figure 1: Sample Health monitor

Several related works have previously surveyed specific areas and technologies related to IoT healthcare. An extensive survey is presented in, with focus placed on commercially available solutions, possible applications, and remaining problems. Each topic is considered separately, rather than as part of an overarching system. In data mining, storage, and analysis are considered, with little mention of integration of these into a system. Sensor types are compared in, with some focus placed on communications. However, it is hard to draw an image of a complete system from this paper. Finally, sensing and big data management is considered, with little regard for the network that will support communications.

Therefore, makes a unique contribution in that it identifies all key components of an end-to-end Internet of Things healthcare system and proposes a generic model that could be applied to all IoT-based healthcare systems. This is vital as there are still no known end-to-end systems for remote monitoring of health in the literature.

Focus is placed on sensors for monitoring various health parameters.

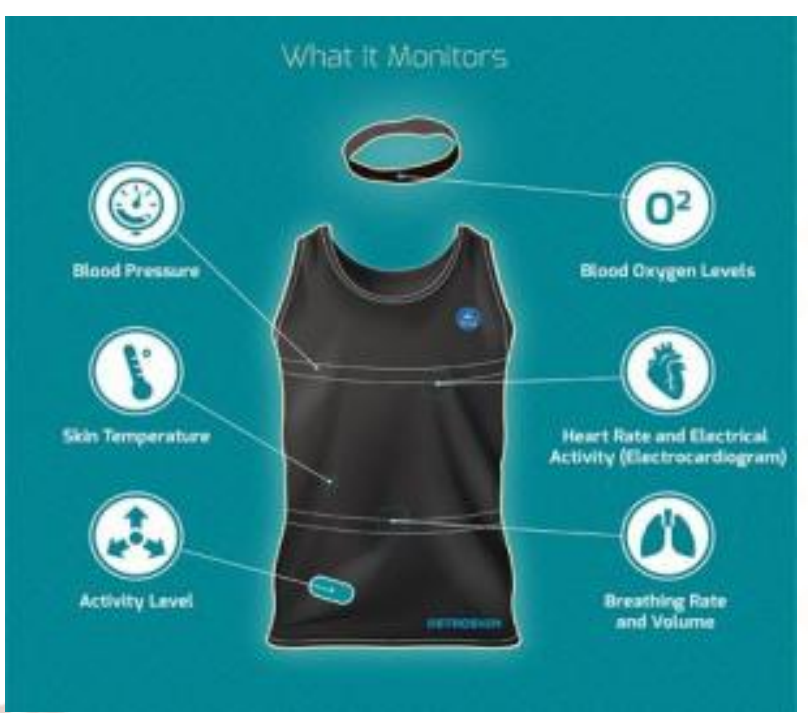

Figure 2: E-textile to monitor health parameter

\section{Use cases of Smart clothing}

Smart clothing has a huge and variety range of applications. The smart clothing can be used in many fields as desired. It can be for personal use or can be used for business/commercial/professional applications.

Few applications or use cases of smart clothing are:

- Performance enhancement for Sport and fitness.

- Healthcare for monitoring patient's health parameters.

- Military for establishment of communication and soldier's health status.

\subsection{Athletics}

Smart wearable clothing is a unique approach to wearable fitness, by making tight-fitting clothing items that are filled with sensors in them. These sensors are used to detect your heart rate, breathing rate and muscle activity. The clothes are swayed with micro sensors that can identify which of your muscles are working in particular and transfer this data to a for their personal analysis. Muscle workout, heart rate and breathing are all tracked and provides insights to help you to workout correctly and avoid any injury. This could act as your personal trainer.

What stands out the most in smart clothing system is that we get insights on where exactly we are exerting ourselves and which specific muscles, as opposed to an underlying workout report — which makes the clothes especially great for training on weight and 
resistance. The users of smart garments have insights to monitor their heart rates, travelled distance, and number of calories burnt while working out/exercising. Few available smart devices such as Fitbits and other healthy lifestyle monitoring devices, connect to the smartphone apps that gives you complete analysis and report on your health, and guidelines to make improve your body health.

Likewise, the development can be made for new smart clothing that can be used/worn every day. Sensors in smart shirts and jackets can tell us many things about our bodies. Temperature, moisture levels, and muscle stress can all be monitored helping us take steps to correct issues before they become a real problem.

You don't have to wear the same dirty clothes every time you work out. The entire clothing features small sensors into cloth, and it connects with the sensors in each item of clothing. The core then wirelessly delivers data to your data analyst.

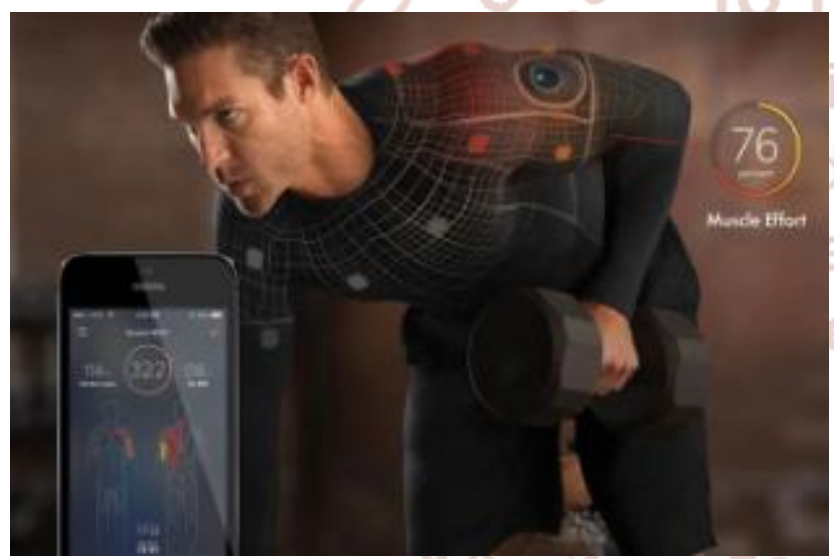

Figure 3: Athlete wearing e-textile

\subsection{Military}

A soldier's life is filled with lot of danger and hazardous situation which they face in their daily life. They overcome these situations by their own intelligence and hard work. The weapons and gadgets which they use will protect and save them in life threatening conditions. These are controlled by the soldier intelligence. The Smart clothing aims at monitoring their health status and try to establish communication among soldiers during danger.

Many soldier's life goes into vain due to insufficient monitor of soldier's health and emergency in war field, search camps, border guarding and training period. The purpose of choosing this project is to provide solution to these problems. The smart clothing aims to provide an Artificial Intelligence to the uniform of soldier which shall be smart enough to monitor heart rate and wound formation and continuously communicate this information with control room.

\section{Soldier-unit:}

This unit is placed on the soldier. It has mainly uses

Sensors: These sensors help in finding the health status of the soldiers, we are making use of kinetic sensor, environmental sensor and other pulse rate sensor. These sensors are helpful in measuring the temperature of their body and the pulse rate and any other changes in soldier's health.

\section{Base unit:}

Upon receiving the data of location of the soldier using global positioning system co-ordinates also the status of their health is displayed in the base unit. In this way all the soldier's health can be tracked and monitored by the army official's.

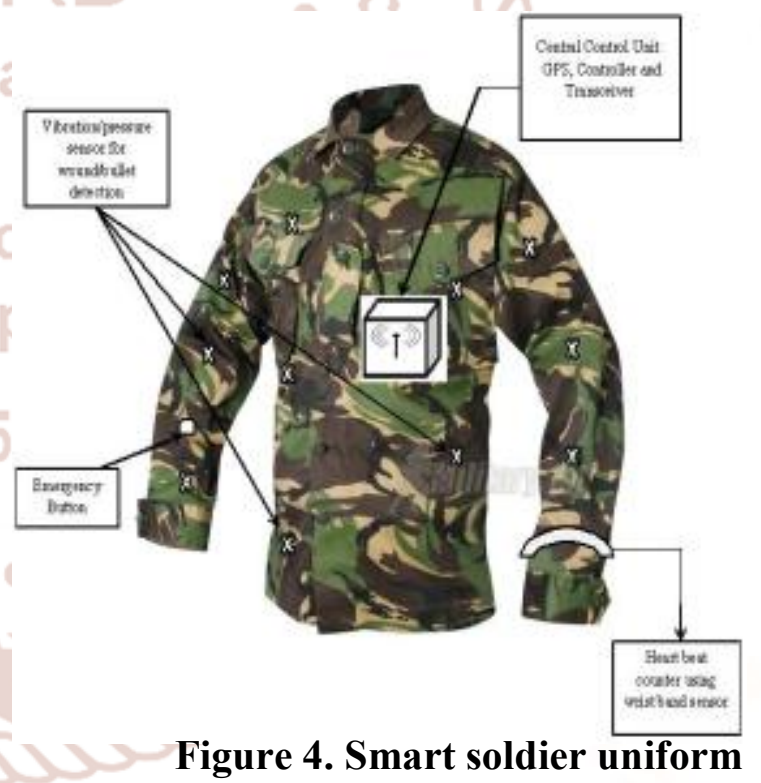

\subsection{Healthcare}

Smart clothing is seen as a way to revolutionize the practice of healthcare, and it's hoped that a widespread use of garments used to monitor health or help with treatment could reduce reliance on costly equipment and a heavily burdened healthcare system. Clothing that can track chronic disease or conditions, help with a growing aging population, or make patients more comfortable during a stay at a hospital or treatment facility is seen as a way to create value, boost health insights, and reduce costs. 
E-textiles are designed to feel comfortable on the skin but at the same time be functional. These smart fabrics consist of traditional fabric woven with conductive fibers as well as electronic elements such as biomedical sensors, microcontrollers, fiber optics and wearable antennas.

They are fully featured, round, sew-able, and Arduino-compatible devices. They are small enough to fit into any project and low cost enough to use without hesitation.

Depending on how the conductive fibers are woven in and the electronics included in the smart clothing, the fabric is durable and able to be washed similar to regular clothing. While durability is still an on-going issue in many projects, it is a consideration that most researchers and companies are working on as an important step toward mass commercialization of smart clothing for healthcare.

\section{Sensors}

In the past few years the sensor technology has developed quite rapidly due to a tremendous escalation of demand in the mobile devices where there is use of nanotechnology with accurate sensors that are multidimensional. This feature rather than making the factor differentiating it has caused hardware issues. This need in sensor technology and the existing challenges have resulted in the advancement for the need of smart clothing. One of the most important developments in sensors and their advancement in technology are miniaturization of sensors, where the cost of production is reduced and the reliability is significantly improved. In the past usage of sensors, the sensors had to be secured/fasten around a particular body part for accurate data measurements. As a result, for a number of years the use of sensors in clothing was completely eliminated as the use of these large sensors lashed around body was uncomfortable and awkward. Another main con of sensors was that they were heavily priced which stopped them to be used vastly for everyday usage. But the requirements and advancements in technology helped in the tremendous growth of sensor technologies. As there is now an availability of micro and nano sensors that are also cost effective and data accurate, they can be used in clothing. Few of the sensors that can be used for smart clothing for the required data measurements would be of physiological sensors, kinetic sensor, and agent or environment detecting sensors.

\subsection{Physiological sensors}

Physiological sensors are the sensors that are used in the measurement of blood-oxygen level, the temperature of body, pressure of blood, composition of urine and sputum. The superior usage of these sensors is to identify the stress level, and injuries.

\subsection{Kinetic sensors}

Kinetic sensors are the type of sensors that are used in the measurement of applied pressure level, movement of muscles, pressure, the number of steps covered by walking/jogging, direction and location using global positioning system. This sensor can be mainly applicable in the field of military as it can be used for the detection of injuries, stress level, and also helps in harmonizing for those on the field and for commanders back at base control room.

\subsection{Environment/agent detection sensors}

Environment/agent detection sensors are the type of sensors that are used in the measurement of atmospheric temperature and pressure, viruses, bacteria and for the detection of chemicals. One of the predominant use of this sensor is to detect danger or emergencies by collecting/reading the data and provide/signal the awareness.

The usage of sensors in smart clothing are gaining its popularity as the sensors are now making use of the micro and nano technologies which fits perfectly in the clothing. These sensors are fixed or embedded as part of the clothing material

\section{Proposed System}

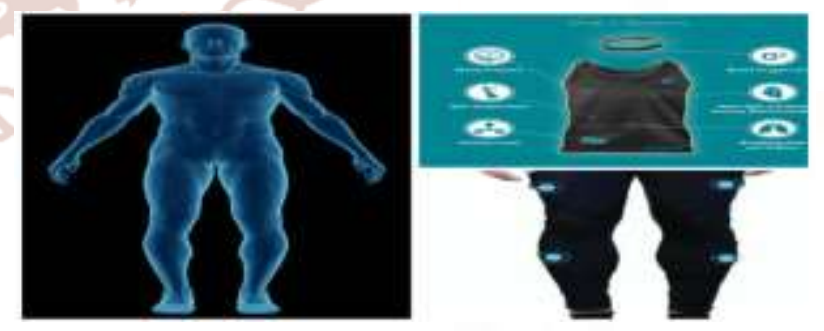

Figure 5: IOT based smart clothing body suit

Due to the ignorance about health this idea has been proposed in order to save the life of common people in the country which can in turn bring the awareness among each one of them which can lead to the healthier and safer side of one's health.

When most people think of health monitoring and analysis in wearable form, they often think of the 
smart watches, fitness monitors, and heart rate monitors that are typically worn on the wrist. However, the smart wearables extend well beyond just these standard devices.

One such emerging market is smart clothing for healthcare--the idea of weaving electronic sensors into a piece of clothing such as shirt, or pants to perform general/specific patient care functions. Clothing that are GPS-embedded for finding children or hikers or skiers when they are lost, and garments that monitor a patient's essential signs.

\section{Advantages}

- Can be implemented in any conditions

- Telemedical records can be stored

- Continuous communication is possible

- Continuous tracking is possible

- Faster communication

- The clothes are washable

- Health status of an individual is monitored

- Carelessness with regards to heath can avoid critical mistakes

- Helps in increasing performance

\section{Challenges for Smart Clothing Market}

As smart clothing market is two-sided one, there are two categories of disadvantages to adapt smart clothing by the common people. From one viewpoint, organizations dispatch items that give individual information to the wearer, then again, collects the big data information that perform business esteem. In this manner, factors, slowing the smart clothing market exist both in favor of organizations and in favor of customers.

There are several problems that are faced by the customers:

\subsection{Lack of awareness about smart clothing}

A Wearable Technology Survey appears smart clothing isn't exceptionally famous with different wearables comparatively. Just $20 \%$ of respondents said that they know about smart clothing. The figures say that prevalence of smart clothing will rise.

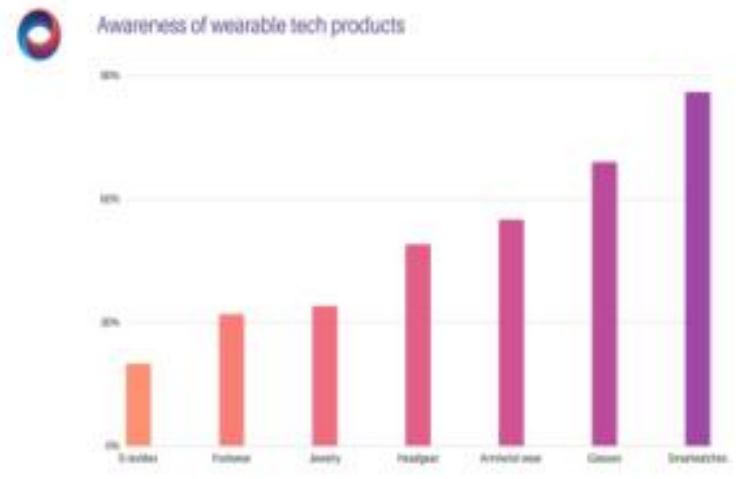

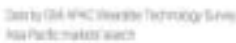

Figure 6: Survey on e-textile awareness

\subsection{Customer worries}

A Wearable Technology Survey shows up, smart clothing isn't particularly renowned in survey with various wearables.

Some of the reasons can be

\subsubsection{Cost}

In the survey of Lightspeed GMI, around $15 \%$ of end-clients guarantee that wearable smart clothing material are excessively costly, making it impossible to purchase. A significant number of respondents are prepared to buy smart clothes if costs is reduced.

\subsubsection{Uselessness}

Around $50 \%$ of potential customers don't know that will wear smart clothing on normal days regularly. Considering the experience of others wearables' utilizing, it is conceivable to expect that shrewd garments is a similar story.

Specifically, surrender rate of smartwatches is $29 \%$ and $30 \%$ for fitness trackers. Such high rates are related with getting exhausted of devices or they break. Uselessness is typically associated with the following customers stress, absence of significant worth.

In similarity with Nielsen's Connected Life Report, in excess of $80 \%$ of respondents said that usefulness is the most vital factor that impacts on making them buy the smart clothes. Under $80 \%$ evaluated comfort as a huge reason. The total rundown of properties that are generally critical for smart clothing is: 
International Journal of Trend in Scientific Research and Development (IJTSRD) ISSN: 2456-6470

- Functionality.

- Comfort.

- Accuracy of data.

- Battery duration.

- Endurance.

\subsubsection{Short-term durability / unreliability}

A long stretch of utilization is a noteworthy factor in the buying of smart clothes since it is furnished with hardware that must be durable while the texture (fiber) must be versatile. One greater prerequisite to smart clothing is the capacity to wash with sensors.

\subsubsection{Personal security threat}

Security of information that wearables give stays topical. Drug, military, and gaming are the principle regions that smart clothing is relevant to. Here information assumes vital part not just for the other party, e. g. Doctor, military administration, group or mentor, yet in addition for the manufacturer of smart clothing. Sensors are attached in smart clothing. These smart clothes track some keep the track of several factors, for example, physical activities, blood glucose level, sleeping habits, are not ensured by Health Insurance Portability and Accountability Act. It implies that such information might be passed to outsiders.

\subsubsection{Not stylish enough}

As Nielsen revealed, around $53 \%$ of extreme clients consider smart clothing is lacking in its style. A few articles of clothing still stay massive and looks too mechanically and not elegant.

\section{CONCLUSION}

IOT can be implemented in many fields of science and technologies one of the common and the most important is healthcare. The result of this domain can be made more efficient in every field by using many trending technologies that has in the recent years. As we mistaken the biggest life taking mistakes by ignoring them considering it to be not so important in one's health. There are many reasons for them to do so which have been mentioned earlier.

Though the idea contains the ongoing glitches but it does contain many other advantages which could be more focused upon. There is a small idea that represents the bright thought of saving the life of Soldiers, Athletes also the common people by proposing the idea of smart clothing which includes sensors in them in order to know the health status of a person like heart rate, blood pressure, muscle fitness, their geographical location during emergency. It can be done by using the different types of sensors and the resources which can keep the track of the person.

Though there exist a lot of challenges in implementation of smart clothing, there are quite a lot advantages of this system and they have wide range of applications.

\section{REFERENCES}

1. A Combination of Internet of Things (IoT) and Graph Database for Future Battlefield Systems Gaurav Tripathi *, Bhawna Sharma*, SonaliRajvanshi* India International Conference on Computing, Communication and Automation (ICCCA2017)

2. Combat triage support using the Internet of Military Things, Proceedings of the Federated Conference on Computer Science and Information Systems pp. 835-842 DOI: 10.15439/2017F186 ISSN 2300-5963 ACSIS, Vol. 11 (2017)

3. Semantic Edge Computing and IoT Architecture for Military Health Services in Battlefield Dhananjay Singh,14th IEEE Annual Consumer Communications \& Networking Conference (CCNC) (2017)

4. Smart Suit for Body Area Network Hiroyuki Arai Department of Electrical and Computer Engineering Yokohama National University, IEEE Asia-Pacific Conference on Applied Electromagnetics (APACE) 11 - 13 December 2016 at Langkawi, Kedah, Malaysia (2016)

5. Smart Questions: Context-dependent mobile information exchange for military operations, 6th International Conference on Knowledge and Smart Technology (KST) (2015)

6. Smart Suit for Body Area Network, Hiroyuki Arai, IEEE Asia-Pacific Conference on Applied Electromagnetics (APACE) 11 - 13 December 2016 at Langkawi, Kedah, Malaysia (2016)

7. Monitoring of Soldier's Health and Transmission of Secret Codes Engr. Zeeshan Raza(2015)

8. Vox, Video and Data Sharing using Body Worn Antennas, Jon Pinto (2015) 